\title{
New Symmetries of the Vacuum Einstein Equations
}

\author{
Metin Gürses \\ Department of Mathematics, Faculty of Science, Bilkent University, 06533 Ankara, Turkey
}

(Received 3 August 1992; revised manuscript received 8 September 1992)

\begin{abstract}
Some new symmetry algebras are found for the vacuum Einstein equations. Among them there exists an infinite-dimensional algebra representing the symmetries analogous to the generalized symmetries of the integrable nonlinear partial differential equations.
\end{abstract}

PACS numbers: $04.20 . \mathrm{Jb}, 04.20 . \mathrm{Cv}, 11.30 . \mathrm{Na}$

In the last decade we observed an increase in the interest on the integrability of the Einstein equations without any spacetime symmetry $[1-15]$. The main purpose of these efforts is to understand up to what degree the Einstein field equations possess the properties of the integrable nonlinear partial differential equations (PDE's). It is now a fact that the tetrad formalism, in particular the $\operatorname{SL}(2, C)$-valued differential forms are more suitable for this purpose. So far it has been shown that the Einstein equations admit nontrivial prolongations [4,5] and Backlund transformations [5,7,9]. In this work we shall show that these equations share another property of the integrable nonlinear PDE's. They admit infinitely many nontrivial symmetries which is a common feature of the integrable nonlinear PDE's. We also show that exponentiating these symmetries leads to formal solutions of the vacuum Einstein equations.

Symmetries and the group invariant solutions of PDE's play an important role in obtaining exact solutions of these equations. They may in general be divided into two parts as the Lie and generalized symmetries. All these symmetries are the solutions of the linearized equations of PDE's under consideration. In particular if a nonlinear PDE has at least one generalized symmetry it is conjectured that this equation is integrable and in this case there is now an algorithm to produce infinitely many of them [16-18]. This leads to the construction of infinitely many conserved quantities and bi-Hamiltonian structures. As an illustration let us give some examples from the Korteweg-de Vries (KdV) equation, $q_{t}=6 q q_{x}$ $+q_{x x x}$. Let $q(t, x, \epsilon)$ be a one-parameter solution of this equation. $\phi=\partial q /\left.\partial \epsilon\right|_{\epsilon=0}$ solves the linearized equation

$$
\phi_{t}=6 q \phi_{x}+6 q_{x} \phi+\phi_{x x x}
$$

A class of solutions of (1) is given by $\phi_{n}=\Phi^{n} q_{x}$ ( $n$ $=1,2, \ldots)$, where $\Phi=D^{2}+4 q+2 q_{x} D^{-1}$ is the recursion operator which transforms symmetries to symmetries. Here $D^{-1} q(t, x)=\int \underline{x}_{\infty} q\left(t, x^{\prime}\right) d x^{\prime}$ and $D q=q_{x}$. These solutions define the following symmetries (flows):

$$
\begin{aligned}
& \delta_{1} q \equiv \phi_{1}=q_{x}, \\
& \delta_{2} q \equiv \phi_{2}=q_{x x x}+6 q q_{x}, \\
& \delta_{3} q \equiv \phi_{3}=q_{x x x x x}+10 q q_{x x x}+20 q_{x} q_{x x}+30 q^{2} q_{x} .
\end{aligned}
$$

All these symmetries commute; $\left[\delta_{m}, \delta_{n}\right]=0$ for all $m$ and $n$. Exponentiating these we obtain group invariant solutions of the $\mathrm{KdV}$ equation. For each $n$ we have different solutions given by $q\left(t, x, \epsilon_{n}\right)=e^{\epsilon_{n} \delta_{n}} q(t, x)$. For $n=1$ and $n=2$ we have the group invariant solutions $q\left(t, x, \epsilon_{1}\right)=q\left(t, x+\epsilon_{1}\right)$ and $q\left(t, x, \epsilon_{2}\right)=q\left(t+\epsilon_{2}, x\right)$ which correspond, respectively, to the translations in the $x$ and $t$ directions. For $n>2$ we have higher symmetries and they do not have closed-form expressions. As a simple example let us consider the first generalized symmetry $(n=3)$ and take $q=-x / 6 t$ as a solution of the $\mathrm{KdV}$ equation. Then we obtain a one-parameter solution as (below we have set and $\epsilon_{3}=6 k / 5$ )

$$
\begin{aligned}
q(t, x, k)= & -\frac{x}{6 t}-k \frac{x^{2}}{6 t^{3}}+k^{2} \frac{2\left(-x^{3}+2 t\right)}{6 t^{5}}+k^{3} \frac{x\left(-5 x^{3}+32 t\right)}{6 t^{7}} \\
& +k^{4} \frac{2 x^{2}\left(-7 x^{3}+96 t\right)}{6 t^{9}}+k^{5} \frac{2\left(-21 x^{6}+512 x^{3} t-392 t^{2}\right)}{6 t^{11}}+\cdots
\end{aligned}
$$

All such formal solutions corresponding to generalized symmetries are infinite series and have no closed form. Formally they are represented as $q(t, x, \epsilon)=e^{\epsilon \delta} q(t, x)$. This way one may also generate multiparameter solutions. Here the order of the operators $e^{\epsilon_{1} \delta_{1}} e^{\epsilon_{2} \delta_{2}} \ldots$ is not important because the operators $\delta_{n}$ commute.

The vacuum field equations in null tetrad formalism are more convenient for our purpose. The $\operatorname{SL}(2, C)$ valued tetrad 1 -form $\sigma$ and $\operatorname{sl}(2, C)$-valued connection 1 form $\Gamma$ are defined as follows:

$$
\sigma=\left(\begin{array}{cc}
n & -m^{*} \\
-m & l
\end{array}\right)
$$

$$
\Gamma=\left(\begin{array}{cc}
\Gamma_{0} & \Gamma_{2} \\
\Gamma_{1} & -\Gamma_{0}
\end{array}\right),
$$

where

$$
\begin{aligned}
& \Gamma_{0}=\gamma l+\epsilon n-\alpha m-\beta m^{*}, \\
& \Gamma_{1}=-\tau l-\kappa n+\rho m+\sigma m^{*}, \\
& \Gamma_{2}=v l+\pi n-\lambda m-\mu m^{*} .
\end{aligned}
$$

Here $l, n, m$, and $m^{*}$ are the null tetrad 1 -forms, $\alpha, \beta, \gamma, \ldots$ are the Newmann-Penrose spin coefficients. An asterisk denotes the complex conjugation. $\Gamma$ and $\sigma$ 
are related through the following equation (definition of the torsionless connection):

$$
d \sigma=-\Gamma \sigma+\sigma \Gamma^{\dagger}
$$

where $†$ denotes the Hermitian conjugation. In this for- malism the vacuum Einstein field equations have the following form:

$$
R \sigma \equiv(d \Gamma+\Gamma \Gamma) \sigma=0 .
$$

Here $R$ is the $\mathrm{sl}(2, C)$-valued curvature 2 -form which is given as $R=\Psi \sigma$ with

$$
\Psi=\left(\begin{array}{cc}
-\Psi_{2}(l-n)-\Psi_{3} m+\Psi_{1} m^{*} & -\Psi_{3}(l-n)-\Psi_{4} m+\Psi_{2} m^{*} \\
\Psi_{1}(l-n)+\Psi_{2} m-\Psi_{0} m^{*} & \Psi_{2}(l-n)+\Psi_{3} m-\Psi_{1} m^{*}
\end{array}\right) .
$$

Here $\Psi_{0}, \Psi_{1}, \ldots, \Psi_{4}$ are the Weyl spinors.

Let the set $\left(\sigma\left(\epsilon_{0}\right), \Gamma\left(\epsilon_{0}\right)\right)$ be a one-parameter solution of the vacuum field equations. Then the set $(t, \omega)$, defined through $\sigma\left(\epsilon_{0}\right)=\sigma+\epsilon_{0} t+\cdots$ and $\Gamma\left(\epsilon_{0}\right)=\Gamma+\epsilon_{0} \omega$ $+\cdots$, satisfy the linearized vacuum equations

$$
D t+\omega \sigma-\sigma \omega^{\dagger}=0, \quad D(\omega \sigma)+R t=0 .
$$

Here $D$ denotes the covariant exterior derivative. We have recently shown that solutions of the above linearized equations play an essential role in obtaining Backlund transformations [5,7]. In this work we show that each solution of these equations leads to a symmetry of the vacuum field equations (5) and (6).

We shall now present some types of symmetries of the vacuum equations. These symmetries are in fact the solutions of the linearized vacuum Einstein equations. We have the following types.

Type (a): The following set $(t, \omega)$ satisfies the linearized vacuum equations; hence it is a symmetry of the vacuum Einstein field equations

$$
t=X \sigma+\sigma X^{\dagger}, \quad \omega=-D X,
$$

where $X$ is an arbitrary traceless $2 \times 2$ matrix and $D$ is the covariant exterior derivative. Let us denote the generator of this infinitesimal transformation as $\delta_{X}$, i.e.,

$$
t=\delta_{X} \sigma, \omega=\delta_{X} \Gamma,
$$

then it is straightforward to show that

$$
\left[\delta_{X}, \delta_{Y}\right]=\delta_{[Y, X]}
$$

This is the local $\operatorname{sl}(2, C)$ algebra. Exponentiating the infinitesimal transformation we obtain

$$
\sigma^{\prime}=\sigma+\epsilon_{0} \delta \sigma+\cdots=e^{\epsilon_{0} X} \sigma e^{\epsilon_{0} X^{\dagger}} .
$$

Such tetrad transformations constitute the gauge group, namely, the group $\operatorname{SL}(2, C)$, of the Einstein theory which leaves the metric unchanged. Hence they do not give us new solutions.

Infinitesimal (coordinate transformations) spacetime symmetries belong to type (a) symmetries [13] but the group structure is different from the local $\operatorname{SL}(2, C)$ because the commutation relation (11) is replaced by $\left[\delta_{X}, \delta_{Y}\right]=\delta_{Z}$ where $Z=\delta_{X} Y-\delta_{Y} X+[Y, X]$. This yields the infinite-dimensional diffeomorphism group.

Type (b): The set $(t, \omega)$ is a symmetry of the vacuum Einstein equations where

$$
t=-D A, \omega \sigma=R A .
$$

Here $A$ is an arbitrary Hermitian $2 \times 2$ matrix. The 1form $\omega$ can be obtained as follows. Let

$$
A=\left(\begin{array}{cc}
a & b \\
b^{*} & c
\end{array}\right)
$$

where $a, c$ are real and $b$ is a complex function. Then

$$
\omega=\left(\begin{array}{cc}
\omega_{0} & \omega_{2} \\
\omega_{1} & -\omega_{0}
\end{array}\right)
$$

where

$$
\begin{aligned}
\omega_{0}= & \left(b \Psi_{1}+c \Psi_{2}\right) n-\left(a \Psi_{2}+b^{*} \Psi_{3}\right) l \\
& -\left(b \Psi_{2}+c \Psi_{3}\right) m+\left(a \Psi_{1}+b^{*} \Psi_{2}\right) m^{*}, \\
\omega_{1}= & -\left(b \Psi_{0}+c \Psi_{1}\right) n+\left(a \Psi_{1}+b^{*} \Psi_{2}\right) l \\
& +\left(b \Psi_{1}+c \Psi_{2}\right) m-\left(a \Psi_{0}+b^{*} \Psi_{1}\right) m^{*}, \\
\omega_{2}= & \left(b \Psi_{2}+c \Psi_{3}\right) n-\left(a \Psi_{3}+b^{*} \Psi_{4}\right) l \\
& -\left(b \Psi_{3}+c \Psi_{4}\right) m+\left(a \Psi_{2}+b^{*} \Psi_{3}\right) m^{*} .
\end{aligned}
$$

The above solution (13) of the vacuum equations defines a symmetry which we denote as $\delta^{A}$. It is given by

$$
\delta^{A} \sigma=-D A, \quad\left(\delta^{A} \Gamma\right) \sigma=R A .
$$

This symmetry is a function of the connection and curvature. The commutator of type (a) and type (b) symmetries gives a type (b) symmetry, i.e.,

$$
\left[\delta^{A}, \delta_{X}\right]=\delta^{C},
$$

where $C=X A+A X^{\dagger}$. Exponentiation of type (b) symmetry does not give a closed-form expression like that of type (a). It reads

$$
\begin{gathered}
\sigma^{\prime}=\sigma-\epsilon_{0} D A-\frac{\epsilon_{0}^{2}}{2 !}\left(\omega A+A \omega^{\dagger}\right) \\
-\frac{\epsilon_{0}^{3}}{3 !}\left(\delta \omega A+A \delta \omega^{\dagger}\right)-\cdots, \\
\Gamma^{\prime}=\Gamma+\epsilon_{0} \omega+\frac{\epsilon_{0}^{2}}{2 !} \delta \omega+\frac{\epsilon_{0}^{3}}{3 !} \delta^{2} \omega+\cdots,
\end{gathered}
$$

where

$$
\begin{aligned}
& (\delta \omega) \sigma=D \omega A+\omega D A \\
& \left(\delta^{2} \omega\right) \sigma=2 \delta \omega D A+(D \delta \omega+3 \omega \omega) A+\omega A \omega^{\dagger} .
\end{aligned}
$$


It is straightforward to calculate each coefficient of $\epsilon_{0}^{n}$ by the use of Eqs. (20) and (21) in an algorithmic way. If the set $(\sigma, \Gamma)$ is a solution of the vacuum equations then the set $\left(\sigma^{\prime}, \Gamma^{\prime}\right)$ given in (18) and (19) is also a solution of the same equations. This is like the exponentiation of generalized symmetries of the $\mathrm{KdV}$ equation.

The commutation relations of two type (b) symmetries give a type (a) symmetry, i.e.,

$$
\left[\delta^{A}, \delta^{B}\right]=\delta_{X}
$$

where

$$
\begin{aligned}
& X=\left(\begin{array}{cc}
-X_{1} & -X_{0} \\
X_{2} & X_{1}
\end{array}\right), \\
& X_{0}=x_{0} \Psi_{0}+x_{1} \Psi_{1}+x_{2} \Psi_{2}, \\
& X_{1}=x_{0} \Psi_{1}+x_{1} \Psi_{2}+x_{2} \Psi_{3}, \\
& X_{2}=x_{0} \Psi_{2}+x_{1} \Psi_{3}+x_{2} \Psi_{4},
\end{aligned}
$$

with

$$
\begin{aligned}
& x_{0}=a_{1} b_{2}-a_{2} b_{1}, \\
& x_{1}=a_{1} b_{3}-a_{3} b_{1}+a_{2}^{*} b_{2}-a_{2} b_{2}^{*}, \\
& x_{2}=b_{3} a_{2}^{*}-a_{3} b_{2}^{*},
\end{aligned}
$$

where

$$
A=\left(\begin{array}{ll}
a_{1} & a_{2} \\
a_{2}^{*} & a_{3}
\end{array}\right), \quad B=\left(\begin{array}{ll}
b_{1} & b_{2} \\
b_{2}^{*} & b_{3}
\end{array}\right) .
$$

The commutation relations in (11), (17), and (22) imply that type (a) and type (b) symmetries together give a closed symmetry algebra of the vacuum Einstein equations in which the local $\mathrm{sl}(2, C)$ symmetry algebra is its maximal subalgebra.

Type (c): The set $\left(t_{1}^{A}, \omega_{1}^{A}\right)$ is a symmetry of the vacuum Einstein equations where

$$
\begin{aligned}
& t_{1}^{A}=-i\left[\Omega^{A} A^{\dagger}-A\left(\Omega^{A}\right)^{\dagger}\right], \\
& \omega_{1}^{A} \sigma=i D\left(\Omega^{A} A^{\dagger}\right)
\end{aligned}
$$

with $\Omega^{A} \sigma=R A$. Here $A$ is a complex $2 \times 2$ matrix. One may exponentiate this symmetry and obtain a formal solution of the vacuum Einstein equations

$$
\begin{aligned}
\sigma^{\prime}= & \sigma-i \epsilon_{0}\left[\Omega^{A} A^{\dagger}-A\left(\Omega^{A}\right)^{\dagger}\right] \\
& -i\left(\epsilon_{0}^{2} / 2\right)\left[\left(\delta \Omega^{A}\right) A^{\dagger}-A\left(\delta \Omega^{A}\right)^{\dagger}\right]-\cdots,
\end{aligned}
$$

where $\delta \Omega^{A}=i \Omega^{A}\left[\Omega^{A} A^{\dagger}-A\left(\Omega^{A}\right)^{\dagger}\right]+\left(D \omega^{A}\right) A$. This type of symmetry provides other new symmetries. For instance, the commutator of type (b) and type (c) symmetries gives a new symmetry of a different type. The commutation relations of this new symmetry with type (b) and type (c) symmetries give other new symmetries. This way one obtains an infinite number of symmetries of vacuum gravitational field equations. This hierarchy of symmetries, as explained above, is obtained through the use of different types of symmetries. On the other hand, there is another type of hierarchy which is obtained by the utility of the same type of symmetry. We shall now give this hierarchy.

It is also possible to generate infinitely many symmetries of the vacuum Einstein equations by the utility of type (c) symmetries. In the following we use lower-case letters $a, b, c, \ldots$ instead of the letters $A, B, C, \ldots$ as the index of type (c) symmetry generators $\delta$. We shall also use a subscript 1 to indicate that $\delta_{1}^{a}$ is the first element of a hierarchy. Let $\left(t_{1}^{a}, \omega_{1}^{a}\right)$ and $\left(t_{1}^{b}, \omega_{1}^{b}\right)$ be symmetries of the vacuum equations of the same type where

$$
\begin{aligned}
& t_{1}^{a} \equiv \delta_{1}^{a} \sigma=-i\left(\Omega^{a} A^{\dagger}-A \Omega^{a^{\dagger}}\right), \\
& \Omega_{1}^{a} \sigma=R A \omega_{1}^{a} \sigma=i D\left(\Omega^{a} A^{\dagger}\right), \\
& t_{1}^{b} \equiv \delta_{1}^{b} \sigma=-i\left(\Omega^{b} B^{\dagger}-B \Omega^{a^{\dagger}}\right), \\
& \Omega_{1}^{b} \sigma=R B \omega_{1}^{b} \sigma=i D\left(\Omega^{b} B^{\dagger}\right) .
\end{aligned}
$$

Here $A$ and $B$ are arbitrary $2 \times 2$ matrices. Then it is easy to show that the new set $\left(t_{2}^{a, b}, \omega_{2}^{a, b}\right)$ satisfies the linearized vacuum equations where

$$
t_{2}^{a, b}=\delta_{1}^{a} t_{1}^{b}-\delta_{1}^{b} t_{1}^{a}, \quad \omega_{2}^{a, b}=\delta_{1}^{a} \omega_{1}^{b}-\delta_{1}^{b} \omega_{1}^{a} .
$$

Denoting $t_{2}^{a, b}=\delta_{2}^{a, b} \sigma$ and $\omega_{2}^{a, b}=\delta_{2}^{a, b} \Gamma$ then we obtain $\delta_{2}^{a, b}=\left[\delta_{1}^{a}, \delta_{1}^{b}\right]$. The new set $\left(t_{2}^{a, b}, \omega_{2}^{a, b}\right)$ is explicitly given by

$$
\begin{aligned}
t_{2}^{a, b} \equiv \delta_{2}^{a, b} \sigma=i\left[\Omega^{a b} B^{\dagger}-B\left(\Omega^{a b}\right)^{\dagger}\right. & \left.-\Omega^{b a} A^{\dagger}+A\left(\Omega^{b a}\right)^{\dagger}\right], \\
& \\
\omega_{2}^{a, b} \equiv \delta_{2}^{a, b} \Gamma, &
\end{aligned}
$$

where $\omega_{2}^{a, b}$ is also found through the following equation [which is in agreement with (30)]:

$$
\begin{aligned}
\omega_{2}^{a, b} \sigma= & i D\left(\Omega^{a b} B^{\dagger}-\Omega^{b a} A^{\dagger}\right)-i \omega_{1}^{b} A \Omega^{a^{\dagger}}, \\
& +i \omega_{1}^{a} B \Omega^{b^{\dagger}}-i \Omega^{b} B^{\dagger} \omega_{1}^{a \dagger}+i \Omega^{a} A^{\dagger} \omega_{1}^{b^{\dagger}} .
\end{aligned}
$$

Here $\Omega^{a b} \sigma=\left(D \omega_{1}^{a}\right) B+i \Omega^{b}\left(\Omega^{a} A^{\dagger}-A \Omega^{a \dagger}\right)$. The new solution $\left(t_{2}^{a, b}, \omega_{2}^{a, b}\right)$ is a function of the connection, curvature, and the first, second, and third derivatives of the curvature. Hence it is different from the first symmetry solution $\left(t_{1}^{a}, \omega_{1}^{a}\right)$. For this reason we use a subscript 2 to indicate that it is a new symmetry.

By the application of $\delta_{1}$ to the new symmetry we obtain another one. For instance, the commutator of $\delta_{1}$ and $\delta_{2}$ gives $\delta_{3}^{a b, c}=\left[\delta_{1}^{a}, \delta_{2}^{b, c}\right]$. Although we do not display it here, the set $\left(t_{3}^{a b, c}, \omega_{3}^{a b, c}\right)$ satisfies the linearized vacuum equations. Hence it is also a new symmetry of the vacuum Einstein equations. In this way we generate infinitely many symmetries $\delta_{n}^{a b c, \ldots, a^{\prime} b^{\prime} c^{\prime}, \ldots}$ with $n=1,2,3, \ldots$. Here the operator $\left[\delta_{1}^{a}, \cdot\right]$ plays the role of recursion operator in the $\mathrm{KdV}$ case. The set $\left(t_{n}, \omega_{n}\right)$ in general depends on the connection, curvature, and the derivatives of the curvature up to the $(n+1)$ th order. The infinitedimensional algebra obtained this way has the following 
commutation relations:

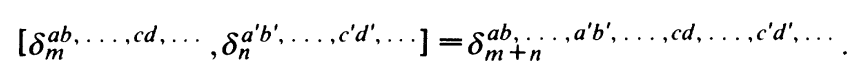

We have verified the Jacobi identities up to $n=4$ and it seems that any set $\left(t_{n}^{a b}, \ldots, c d, \ldots, \omega_{n}^{a b}, \ldots, c d, \ldots\right)$ satisfying the linearized vacuum equations $(8)$, the symmetry equation, the corresponding generators $\delta_{n}^{a b}, \ldots, c d, \ldots$ satisfy the Jacobi identities. We have not been able to identify the above infinite-dimensional algebra yet, but it seems that it is more general than the Kac-Moody, Virasoro, and $W_{\infty}$ algebras.

We have found some new symmetries of the vacuum Einstein field equations. They contain Lie type of symmetries like type (a) and type (a) + type (b) symmetries and also generalized symmetries like type (c).

This work is partially supported by the Scientific and Technical Research Council of Turkey (TUBITAK) under TBAG-ÇG-1.

[1] R. Penrose and W. Rindler, Spinors and Space-Time (Cambridge Univ. Press, Cambridge, 1986), Vol. 2.

[2] B. Julia, C. R. Acad. Sci. Paris, Series 2, 295, 113 (1982).

[3] M. Dubois-Violette, Phys. Lett. 131B, 323 (1982).

[4] F. J. Chinea, Phys. Rev. Lett. 52, 322 (1984).

[5] M. Gürses, Phys. Lett. 101A, 388 (1984).
[6] K. P. Tod, Phys. Rev. Lett. 54, 1594 (1985).

[7] A. Bilge and M. Gürses, J. Math. Phys. 22, 1319 (1986).

[8] B. K. Harrison, in Proceedings of the Fourth Marcel Grossmann Meeting on General Relativity, edited by R. Ruffini (Elsevier, Amsterdam, 1986).

[9] M. Gürses and A. H. Bilge, in Proceedings of the Fourth Marcel Grossmann Meeting on General Relativity (Ref. [8]).

[10] M. Gürses, in Proceedings of the International Conference on Differential Geometric Methods in Theoretical Physics, edited by G. Doebner and T. D. Palev (World Scientific, Singapore, 1986).

[11] F. B. Estabrook, in Proceedings of the Fourteenth Yamada Conference on Gravitational Collapse and Relativity, edited by $\mathrm{H}$. Sato and T. Nakamura (World Scientific, Singapore, 1987).

[12] M. Gürses, in Proceedings of the Fourteenth Yamada Conference on Gravitational Collapse and Relativity (Ref. [11]).

[13] F. J. Chinea, Classical Quantum Gravity 5, 135 (1987).

[14] F. B. Estabrook, Acta Aplicanta Mathematicia 8, 293 (1987).

[15] M. Gürses, Lett. Math. Phys. 17, 231 (1989).

[16] A. S. Fokas, SIAM 77, 253 (1987).

[17] A. V. Mikhailov, A. B. Shabat, and V. V. Sokolov, in What is Integrability, edited by V. E. Zakharov (Springer-Verlag, Berlin, 1991).

[18] M. Gürses, A. Karasu, and A. Satir, in Nonlinear Evolution Equations and Dynamical Systems, edited by $\mathbf{M}$. Boiti, L. Martina, and F. Pempinelli (World Scientific, Singapore, 1992). 